\title{
¿Cómo perciben los adolescentes de la ciudad de Buenos Aires su futuro? Una mirada desde sus actores*
}

\section{How adolescents from Buenos Aires city perceive their future? An approach from its actors}

\author{
María Fernanda Molina** \\ ORCID 0000-0002-7361-7058 \\ Consejo Nacional de Investigaciones \\ Científicas y Técnicas (CONICET) \\ Universidad de Buenos Aires
}

Mariel Giménez

Secretaría de Integración Social y Urbana de la Ciudad Autónoma de Buenos Aires

María Julia Raimundi ORCID 0000-0002-4677-3987

Consejo Nacional de Investigaciones

Científicas y Técnicas (CONICET)

Universidad de Buenos Aires

Nora Leibovich de Figuero

Consejo Nacional de Investigaciones

Científicas y Técnicas (CONICET)

Universidad de Buenos Aires

Vanina Schmidt

ORCID 0000-0003-3239-4043

Consejo Nacional de Investigaciones

Científicas y Técnicas (CONICET) Universidad de Buenos Aires

Recibido: 7 de octubre de 2016 Revisado: 18 de marzo de 2017 Aceptado: 3 de mayo de 2017

\section{Resumen}

La forma en que los adolescentes perciben su futuro tiene una gran importancia en su desarrollo positivo. Este trabajo se propone conocer y comprender cómo un grupo de adolescentes escolarizados de la Ciudad Autónoma de Buenos Aires percibe su futuro. Se realizó un estudio de tipo exploratorio-descriptivo con metodología cualitativa y un diseño transversal. Se trabajó con una muestra intencional de 24 adolescentes $(M=16.10 ; D E=1.10)$ de ambos sexos (mujeres $=54,20 \%$ ). Se realizaron entrevistas individuales y grupos focales. Se realizó un análisis de contenido temático con los datos recolectados. Se encontraron dos grandes temas respecto de la percepción del futuro en los adolescentes. El primero se refiere a la imagen que los adolescentes tienen de su futuro. Sus componentes son: valoración, contenido y grado de claridad de la imagen del futuro. El segundo hace referencia a la forma en que los adolescentes construyen su futuro. Sus componentes son: ocuparse del futuro, entornos en los que los adolescentes construyen su futuro, influencia de la familia y valoración del proyecto. Un hallazgo emergente es que los

Artículo de investigación. Este trabajo fue financiado por la Secretaría de Ciencia y Técnica de la Universidad de Buenos Aires. Proyecto uBAcyT 20020130100434BA. Experiencias subjetivas positivas en músicos y deportistas. Su relación con variables de personalidad y contextuales. Directora: Vanina Schmidt. Co-directora: Dra. Nora Leibovich de Figueroa. Proyecto subsidiado. Resolución CS n: 921/14. Agradecimientos: se agradece a los alumnos, docentes, y autoridades de las escuelas en las que se realizó el estudio, por su colaboración en este proyecto de investigación. DOI: http://dx.doi.org/10.15332/s1794-9998.2017.0002.08

** Correspondencia: Dra. María Fernanda Molina: fernandamolina@psi.uba.ar. Dirección postal: Gral. Juan Lavalle 2353. Ciudad Autónoma de Buenos Aires (C.A.B.A.), Argentina. (C.P.: C1052AAA). 
adolescentes difieren en la descripción que hacen de ellos mismos, de sus pares cercanos o de los otros adolescentes en general.

Palabras clave: adolescencia, estudio cualitativo, futuro, percepción.

\begin{abstract}
The way adolescents perceive their future is very important for a positive development. This work aims to identify and understand how a group of adolescent students form Autonomous City of Buenos Aires perceive their future. An exploratory-descriptive study with qualitative methodology and crosssectional design was conducted. We worked with a purposive sample of 24 adolescents $(M=16.10$, $S D=1.10$ ) of both sexes (female $=54.20 \%$ ). Individual interviews and focus groups were conducted. Thematic content analysis of data collected was performed. Two main themes were found regarding adolescents' perception of their future. The first concerns the view adolescents have about their future. Its components are: evaluation, content and degree of image clarity of the future. The second concerns the way adolescents build their future. Its components are: dealing with the future, environments where adolescents build their future, family influence, and evaluation of the project. An emerging finding is that adolescents differ in their descriptions made of themselves, of their closest peers or from other adolescents in general.
\end{abstract}

Keywords: adolescence, qualitative study, future, perception.

El estudio de la visión que los adolescentes tienen de su futuro cobra fundamental interés, pues "como ninguna otra etapa vital, la adolescencia es un período de posibilidad y de las promesas y preocupaciones asociadas a esa posibilidad" (Oyserman \& Markus, 1990, p.112). En la adolescencia se desarrolla la capacidad de pensar, soñar y planear el futuro (Blakemore \& Choudhury, 2006). Es un período de exploración en el que la planificación del futuro emerge como una tarea importante para el desarrollo evolutivo (Erikson, 1968).

Existen diferentes perspectivas teóricas que se han ocupado del estudio de la proyección que hacen los adolescentes hacia el futuro. Una de ellas es la Perspectiva de Tiempo Futuro (PTF). Este constructo es definido como el grado y la forma en que el futuro cronológico es integrado en el espacio de vida presente de un individuo mediante procesos motivacionales de establecimiento de metas (Stouthard \& Peetsma, 1999). Esta teoría hace énfasis en la habilidad cognitiva de las personas para anticipar las consecuencias futuras, inmediatas y en el largo plazo, de una tarea (Phan, 2009).
A partir de una revisión de diversos trabajos, Husman y Shell (2008) señalan que la perspectiva de tiempo futuro constituye un conjunto de creencias relativamente estables y adquiridas con relación al futuro, y que tienen sus raíces en el contexto social cultural. Los autores proponen cuatro dimensiones: el valor o importancia de la meta futura; la extensión, cuán lejos las personas pueden proyectarse en el tiempo con su pensamiento; la velocidad con que pasa el tiempo; y la conexión, es decir, la habilidad para poder conectar las actividades presentes con las metas futuras.

De forma similar, Nurmi (1991) propone que la orientación de los adolescentes al futuro involucra una serie de procesos: la motivación, en el sentido del desarrollo de metas mediante la comparación de los propios motivos y valores con las expectativas que se tienen del futuro; la planificación y la evaluación de la posibilidad de alcanzar la meta y concretar los planes que se construyeron.

También en la adolescencia se construye el sí mismo en el que uno se podría convertir (Erikson, 
1968). En la adolescencia temprana, los jóvenes empiezan a utilizar descripciones más abstractas y pueden integrar diferentes perspectivas del sí mismo: cómo son, cómo fueron, cómo quieren ser, y cómo se presentan al mundo (Oyserman, 2001). Una de las tareas centrales en esta etapa es la elaboración de la imagen del adulto que los adolescentes podrían ser (Erikson, 1968).

Otra de las perspectivas teóricas que se ocupan de la percepción del futuro en los adolescentes es la que estudia los posibles sí mismos. Estos constituyen el autoconocimiento que hace referencia al propio potencial y futuro (Markus \& Nurius, 1986). Son los componentes multifacéticos del autoconcepto que se encuentran proyectados hacia el futuro (Oyserman \& Fryberg, 2006). Pueden hacer referencia al sí mismo en el que la persona cree que se va a convertir, en quién teme convertirse o quién desea ser (Markus \& Nurius, 1986). No son simplemente las metas o aspiraciones del individuo, sino que el adolescente tiene que percibirse como agente de esa situación, experimentándola desde el interior (Erikson, 2007). Es decir, no es lo mismo aspirar a estudiar una carrera universitaria que imaginarse a uno mismo como estudiante o desempeñando una profesión.

Las diferentes perspectivas teóricas presentadas anteriormente señalan la importancia que tiene la percepción del futuro para un desarrollo positivo en los adolescentes. Principalmente, hacen hincapié en su carácter motivacional (e.g. Husman \& Lens, 1999; Markus \& Nurius, 1986). Una perspectiva futura positiva en relación a una meta puede llevar a que la persona se desempeñe mejor para poder alcanzarla (Husman \& Lens, 1999). El sentido de propósito para el futuro cumple un importante rol en la motivación para comprometerse en actividades que podrían derivar en logros futuros (Mclnerney, 2004), contribuye a que el individuo pueda postergar gratificaciones presentes en función de obtener beneficios futuros $\mathrm{y}$, de esta forma, contribuye a la autorregulación del comportamiento (Bembenutty \& Karabenick, 2004; Miller \& Brickman, 2004).

Los posibles sí mismos, proveen un nexo entre el autoconcepto actual y la motivación, y cumplen dos funciones principales. En primer lugar, en tanto proveen al individuo de una representación de cómo desea o teme ser en el futuro, dan sentido a los comportamientos nuevos, los motivan, enmarcan y guían su curso. En segundo lugar, proveen un marco evaluativo e interpretativo para la actual visión del sí mismo, en tanto esta se acerca o no a la representación del sí mismo que se aspira a alcanzar (Markus \& Nurius, 1986).

La importancia que tiene el futuro para el desarrollo de los adolescentes abona al interés de sondear las creencias que estos tienen en relación al tiempo futuro, e insta a intentar comprender cómo se constituyen dichas creencias en el nicho contextual en el que el adolescente se desarrolla.

La perspectiva de tiempo futuro se desarrolla en un contexto sociocultural. Como un producto de la socialización, los individuos aprenden cuáles son las metas que su clase social, su cultura y la sociedad en general espera de ellos (Phan, 2009). Muchos factores pueden influenciar la extensión temporal en la que los individuos pueden proyectar a futuro: la complejidad y los valores de la sociedad en que vive el individuo, las oportunidades percibidas que tienen que ser planificadas en ella, la influencia parental, la tecnología, la espiritualidad (Mclnerney, 2004).

También la naturaleza del futuro que los jóvenes pueden articular para ellos está influida por el contexto sociocultural y la época (Mclnerney, 2004). El funcionamiento de la sociedad actual, su diversificación y complejización llevó a la modificación de los trayectos de vida que antes podían preverse en sus distintas etapas vitales y las metas asociadas a ellas (Krauskopf, 2003). Nuestro mundo es rápidamente cambiante. Las generaciones pasadas tenían un futuro relativamente predecible. En este mundo cambiante surge el interrogante de cuánto de este futuro predecible persiste para los jóvenes de hoy, y en qué medida estos cambios y la falta de certeza afectan su perspectiva sobre el futuro (Mclnerney, 2004).

El contexto social también tiene una fuerte influencia en la constitución de los posibles sí mismos. Un individuo puede crear una variedad de posibles sí mismos. Sin embargo, el grupo de posibles 
sí mismos de que dispone una persona deriva de las categorías salientes en el contexto sociocultural e histórico (Markus \& Nurius, 1986). Lo que las personas importantes para el adolescente esperan de él, lo que piensan que él debería ser, también tiene una fuerte influencia en los posibles sí mismos que desarrolle. El entorno social provee feedback de si un posible sí mismo es valioso o no (Oyserman \& Fryberg, 2006).

Teniendo en cuenta la influencia del entorno social y cultural en la percepción del futuro y los cambios ocurridos en los últimos tiempos respecto de las trayectorias de vida posibles, surge el interrogante sobre cómo los adolescentes de nuestro contexto perciben su futuro y cómo se perciben a ellos mismos en él. Al conocer la construcción sociocultural del futuro, interesa conocer las concepciones de los propios adolescentes acerca de su futuro desde su propia voz. Es por esto que el presente artículo se constituye como un estudio de tipo exploratorio en el que se buscó conocer y comprender como un grupo de adolescentes escolarizados de la Ciudad Autónoma de Buenos Aires (CABA) percibe su futuro, cómo se perciben a ellos mismos en él y qué lugar le dan al futuro en su vida presente.

\section{Método}

Este artículo se considera como de tipo exploratorio-descriptivo, en tanto el interés estuvo centrado en identificar y comprender los sentidos y conceptualizaciones que los adolescentes, en tanto actores sociales, poseen acerca de su futuro. Con este fin, se adoptó una metodología de tipo cualitativa y un diseño transversal. Se utilizó el procedimiento de análisis de la contenido temático (Strauss \& Corbin, 1990).

\section{Participantes}

Se trabajó con una muestra intencional de adolescentes $(n=24)$ de ambos sexos (mujeres $=54,20 \%$ ) que asistían al tercer año de dos escuelas públicas de educación media de la CABA. Las edades de los adolescentes oscilaban entre los 15 y 17 años $(M=$ 16.10; $D E=1.10$ ).
La muestra fue delimitada en función de criterios considerados como relevantes (ser adolescente de sexo femenino o masculino y estar cursando el tercer año de una escuela pública de la CABA) según los objetivos del presente trabajo. Para esto se seleccionaron contextos y personas que puedan brindar la información necesaria para responder a los objetivos propuestos. El propósito de dicha selección no es la representatividad sino la posibilidad de profundizar el sentido de los fenómenos sociales en un determinado grupo (Maxwell, 2012).

\section{Técnicas de producción de la información}

Entrevistas individuales: se realizaron cuatro entrevistas individuales a adolescentes de ambos sexos, con las siguientes preguntas: ¿cómo piensan el futuro los adolescentes?, ¿cómo imaginas el futuro de los chicos de tu su edad?, ¿tu curso en general?, ¿cómo te imaginas en el futuro?, ¿cómo desearías que fuera?

Grupos focales: se realizaron tres grupos focales: uno masculino y otro femenino por colegio, y un grupo mixto en una de las escuelas. Los grupos fueron de cinco a ocho integrantes y tuvieron aproximadamente una hora de duración. Los temas fueron los mismos que en las entrevistas pero el objetivo fue indagar el sentido dado desde una elaboración grupal. Asimismo, la utilización de otra técnica de producción de los datos, posibilita la triangulación de la información, que permite otorgar credibilidad y validez al estudio.

\section{Análisis de los datos}

Se utilizó el método de análisis de contenido temático para el trabajo con el material discursivo.

En primer lugar, se trabajaron las grabaciones de todo el material recolectado (entrevistas y grupos focales), se transcribió tanto lo comunicado verbalmente como lo no verbal.

Los tres integrantes del equipo de investigación recibieron una copia de todo el material y, de modo 
independiente, cada uno identificó unidades de significado relevantes para el objetivo de investigación asignándoles un código preliminar (codificación abierta).

En segundo lugar, los integrantes del equipo presentaron la codificación realizada y se comparó la selección de las unidades de significado y la descripción de categorías realizada por cada uno. Se mantuvieron aquellas categorías que resultaron fáciles de inferir y aplicar, con relevancia teórica, y resultantes del consenso entre los investigadores.

Luego de este proceso fue necesario desarrollar reglas explícitas para lectura del material a partir del establecimiento de códigos, por lo que se creó un libro de códigos. De esta forma quedó explicitado cómo segmentar todo el corpus. Con base en las categorías propuestas en el proceso anterior, se confeccionaron fichas de análisis en las que se consignó una palabra clave o un juicio extraído del texto en su literalidad.

En último lugar, como criterios de validez en metodología cualitativa, un investigador, externo al proceso ya realizado, fue convocado para revisar y auditar las categorizaciones realizadas. El investigador actuó como "procesador de datos", trabajó con fichas de análisis (Piñuel, 2002) y procedimiento que se orientó a establecer la "credibilidad", "dependencia" y "confirmabilidad" de los datos (Hernández, Fernández-Collado y Baptista, 2010).

\section{Resultados}

A continuación se describen los temas y categorías encontradas y consensuadas. En muchas de estas categorías se encontró que los adolescentes difieren en la descripción que hacen de ellos mismos, de sus pares más cercanos o de los otros adolescentes en general. Esta diferenciación se presentará a lo largo de la descripción de las categorías y se resume en la tabla 1.

\section{Tema I. Imagen del futuro}

El primer tema encontrado se refiere a la imagen del futuro que tienen los adolescentes, a cómo visualizan ellos su futuro. Sus componentes son: valoración, contenido y grado de claridad de la imagen del futuro.

I.a. Valoración. La imagen del futuro puede ser positiva o negativa. Los adolescentes se perciben a sí mismos, en el futuro, de manera positiva.

"Y... yo me veo bien, la verdad es que bien. Me veo en lo que me gusta, que es lo maternoinfantil. Yo me veo bien." [Entrevista individual, femenina]

A sus pares cercanos también los ven de manera positiva.

"A la mayoría los veo que van a seguir el colegio y que se van a interesar en ser alguien y hacer algo el día de mañana." [Entrevista individual, femenina]

En cambio, el futuro de los otros adolescentes es negativo.

Adolescente 1: "Mal si hacen las cosas que dijimos, si se están drogando." Adolescente 2: "Van a terminar mal." [Grupo focal, femenino]

I.b. Contenido. Cuando se refieren a sí mismos, el contenido de la imagen del futuro de los adolescentes se relaciona con el estudio - tanto terminar la escuela como seguir estudiando después- el trabajo, la familia, y la propia independencia y autosustento.

"Me imagino terminando la secundaria, estudiando algo..." [Entrevista individual, femenina]

"Yo, mi futuro, por lo menos lo que tengo planeado yyy... termino el colegio y me voy a La Plata a estudiar penitenciaría. Y termino eso y empiezo un profesorado de geografía (...) para... por lo menos, si algún día tengo una familia, tenerla bien." [Entrevista individual, masculina]

El futuro de los pares se ve de manera similar al propio: terminar el secundario, poder sustentar una familia y tener un trabajo que les guste. 
Tabla 1.

Comparación en la percepción del futuro para sí mismo, sus pares y los otros adolescentes

\begin{tabular}{|c|c|c|c|c|}
\hline Tema & Categoría & Sí mismo & Nosotros & Los otros \\
\hline \multirow{3}{*}{ 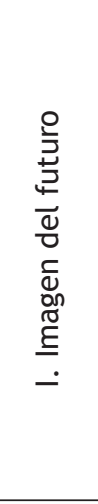 } & I.a. Valoración & Positiva & Positiva & Negativa \\
\hline & I.b. Contenido & $\begin{array}{l}\text { El estudio, el trabajo, la } \\
\text { familia, y la propia inde- } \\
\text { pendencia y autosustento. }\end{array}$ & $\begin{array}{l}\text { Terminar el secundario, } \\
\text { poder sustentar una fa- } \\
\text { milia y tener un trabajo } \\
\text { que les guste. }\end{array}$ & $\begin{array}{l}\text { Van a terminar mal, } \\
\text { muertos, adictos, "no } \\
\text { van a ser nada". }\end{array}$ \\
\hline & I.c. Claridad & $\begin{array}{l}\text { Algunos tienen un } \\
\text { proyecto claro para el } \\
\text { que ya están trabajando, } \\
\text { otros tienen una idea que } \\
\text { no saben si van a poder } \\
\text { lograr, otros refieren que } \\
\text { sus metas y proyectos van } \\
\text { cambiando. }\end{array}$ & $N / R$ & $N / R$ \\
\hline \multirow{4}{*}{ 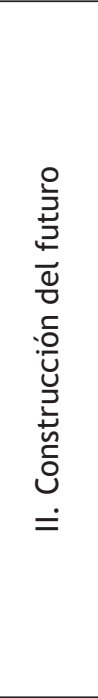 } & $\begin{array}{l}\text { Il.a. "ocuparse del } \\
\text { futuro" }\end{array}$ & $\begin{array}{l}\text { Anticipan, piensan y plani- } \\
\text { fican su futuro. }\end{array}$ & $N / R$ & $\begin{array}{l}\text { No piensan en el futuro, } \\
\text { no lo planifican, ni } \\
\text { anticipan las consecuen- } \\
\text { cias de sus acciones. No } \\
\text { trabajan para el futuro, } \\
\text { están centrados en el } \\
\text { ahora. }\end{array}$ \\
\hline & $\begin{array}{l}\text { Il.b. Entornos en los que } \\
\text { los adolescentes constru- } \\
\text { yen su futuro }\end{array}$ & $\begin{array}{l}\text { Dan importancia a la } \\
\text { escuela y a las actividades } \\
\text { extracurriculares para la } \\
\text { construcción de su futuro. }\end{array}$ & $N / R$ & $\begin{array}{l}\text { No tienen en cuenta a la } \\
\text { escuela como una herra- } \\
\text { mienta para su futuro. } \\
\text { No le dan importancia, } \\
\text { no estudian, repiten. }\end{array}$ \\
\hline & $\begin{array}{l}\text { II.c. Influencia de la } \\
\text { familia }\end{array}$ & $\begin{array}{l}\text { La familia participa en la } \\
\text { construcción de futuro. }\end{array}$ & $N / R$ & $N / R$ \\
\hline & $\begin{array}{l}\text { II.d. Valoración del } \\
\text { proyecto }\end{array}$ & $\begin{array}{l}\text { Las características del } \\
\text { proyecto también influyen } \\
\text { en la construcción del } \\
\text { futuro. }\end{array}$ & $N / R$ & $N / R$ \\
\hline
\end{tabular}

Nota. N/F = no hacen referencia

"A la mayoría los veo que van a seguir el colegio y que se van a interesar en ser alguien y hacer algo el día de mañana (...) En el día de mañana poder llevar adelante una familia y tener una base, poder hacer algo y no terminar haciendo cosas que a uno no le gustan para poder tener plata." [Entrevista individual, femenina]

El contenido de la imagen del futuro de los otros adolescentes involucra "terminar mal", "no ser nada", la adicción a las drogas o, incluso, la muerte.

Adolescente 1: "Terminar muerto". Adolescente 2: "Ser adicto." Adolescente 3: "Y si no van a la escuela, si no se preocupan no van a ser nada." [Grupo focal, femenino]

I.c. Grado de claridad de la imagen en el futuro. Los adolescentes difieren en el grado de claridad y confianza con el que pueden proyectar una imagen de su futuro. Algunos tienen un proyecto claro para el que ya están trabajando, otros tienen una idea que no saben si van a poder lograr, otros refieren que sus metas y proyectos van cambiando.

"Entrevistador: ¿qué es lo que te gustaría hacer? Adolescente: traductora... Entrevistador: ¿Hace cuánto estudiás? Adolescente: hace cinco años. Entrevistador: ¿y ya pensaste 
dónde vas a estudiar? Adolescente: sí, sí... ya cuando termine acá, ya sé qué universidad." [Entrevista individual, femenina]

“...Yo, o sea, yo tengo una guía planeada pero no sé si va a ser así. Yo me pongo a pensar, tengo lo que qui... tengo un plan, tengo en mente lo que quiero estudiar, tengo en mente lo que quiero hacer perooo no sé si voy a llegar..." [Entrevista individual, masculina]

“...al principio quería ser médico, porque me gustaba, me llamaba... Después me empezó a llamar por la computación, más por la tecnología, y ahora prefiero ser Técnico reparador. Vaya a saber en quinto qué quiero ser..." [Adolescente varón, grupo focal mixto escuela 31]

Esta categoría solo apareció en relación al sí mismo y no con respecto al grupo de pares o a los otros adolescentes.

\section{Tema II. Construcción del futuro}

El segundo tema encontrado hace referencia a la forma en que los adolescentes construyen su futuro. Sus componentes son: ocuparse del futuro, entornos en los que los adolescentes construyen su futuro, influencia de la familia, valoración del proyecto.

II.a. Ocuparse del futuro. La primera categoría se refiere a las acciones que realizan los adolescentes en el presente para construir su futuro.

Los adolescentes se ocupan del futuro cuando lo planifican, lo anticipan y toman decisiones en el presente, pensando en lograr esos objetivos. Elegir qué es lo que uno quiere hacer y tenerlo como horizonte. Saber qué es lo que quieren hacer cuando terminen el secundario es un aspecto importante de la planificación de su futuro.

"Y... por lo menos desde que entraste a la secundaria, ya saber más o menos lo que querés hacer. Por lo menos en tercer año ya saber si querés un comercial, un bachiller, tener una orientación de qué vas a querer seguir después, de qué estudio vas a querer recibirte (...) A una temprana edad que te plantees bien lo que querés hacer, para después ya estar más o menos ubicado" [Entrevista individual, femenina].

Desde la percepción de los adolescentes entrevistados, los "otros" adolescentes no piensan en el futuro, no lo planifican, ni anticipan las consecuencias de sus acciones. No trabajan para el futuro, están centrados en el ahora, viven el presente y van a decidir después. Solo algunos adolescentes se ocupan de su futuro, es decir, estudian y piensan en lo que quieren hacer. Los otros adolescentes no tienen un plan o metas establecidas.

“...yo creo que ahora, no se esmeran mucho los adolescentes. No se esmeran en pensar en un futuro ni estudiar, sino que la mayoría hacen la suya, estudian cuando tienen ganas y los fines de semana son para salir a bailar... cómo te puedo explicar... a divertirse directamente (...) viven el presente y cuando tenga que llegar, creen que van a saber qué hacer." [Entrevista individual, femenina]

“Mmmm... ¿Cómo piensan el futuro?... No, no hay muchos que piensan el futuro. No hay muchos que se pongan a pensar que van a hacer el día de mañana (...) no tienen una meta, un, un norte a seguir, no tienen nada. Viven el día a día y no piensan en lo que va a pasar mañana." [Entrevista individual, masculina]

Los adolescentes perciben diferencia de género con relación a la percepción del futuro y del presente. Sostienen que hay una mayor madurez en las mujeres. También en este caso pensar en el futuro es asociado con el estudio y el compromiso con la escuela. Se percibe que las mujeres piensan más en el futuro porque se ocupan más de la escuela. Los otros varones no piensan en el futuro o no demuestran pensar en ello en tanto no se ocupan de la escuela.

"Las chicas son más maduras... en cambio los pibes nah, son vagancia. La mayoría... nadie piensa en el futuro. $Y$ si piensan no lo demuestran. [Las chicas] se ríen de lo que hacen los pibes pero no hacen lo que hacen los pibes... Se centran más en la parte escolar, o sea, está explicando el profesor y no están 
cagándose de risa, jugando a las cartas o escuchando música. La mayoría, la mayoría están escuchando lo que está diciendo." [Entrevista individual, masculina]

\section{II.b. Entornos en los que los adolescentes cons-} truyen su futuro. El tipo de participación que tienen los adolescentes en algunos de los entornos en los que se desenvuelven es central para la construcción de su futuro.

La escuela aparece como uno de los contextos principales en los que los adolescentes construyen su futuro. La escuela, en general, se percibe como una herramienta para poder seguir estudiando (e.g. en la universidad) y elegir mejores trabajos.

"Entrevistador: y los que dijeron que no dejarían la escuela, ¿por qué seguirían?, ¿cuáles son las ventajas de seguir?" Adolescente 1: "Yo porque quiero estudiar." Adolescente 2: "porque quiero llegar a ser alguien. Tener metas." [Grupo focal masculino]

También el estudio en sí mismo es percibido como una herramienta para el futuro. Lo vinculan con la posibilidad de progreso y de trabajos "más cómodos”. Hay salidas más rápidas para obtener dinero, pero estancan el desarrollo. "Si dejás la escuela te vas a arruinar la vida". Una vez que esta etapa termina es irreversible: "quemás la etapa".

“Todo se piensa cuando termine el colegio. Después, ahí quemás una etapa total... acá está la formación, la escuela es la formación. Te va formando para poder llegar, o sea depende de la formación es a lo que vas a poder aspirar..." [Entrevista individual, masculina]

También las actividades extracurriculares aparecen como un espacio en el que los adolescentes pueden construir su futuro empezando la formación para la profesión que quieren seguir después o aprender un oficio.

"Estoy haciendo este curso de electricidad para, no sé, ya empezar algo, porque sino no sé (...) esto me va a dar trabajo el día de mañana...." [Grupo focal masculino]
Desde la percepción de los participantes, los otros adolescentes no le dan importancia a la escuela, no estudian, repiten, no la tienen en cuenta como una herramienta importante para su futuro.

"y yo creo que ahora, no se esmeran mucho los adolescentes. No se esmeran en pensar en un futuro ni estudiar, sino que la mayoría hacen la suya, estudian cuando tienen ganas y los fines de semana son para salir a bailar...cómo te puedo explicar...a divertirse directamente (...) creo que hay entre cinco y diez que repitieron. Así que, yo espero que se pongan las pilas." [Entrevista individual, femenina]

"El colegio es un embole, esa es la posta, pero... Si lo pensás 2 veces te vas a arruinar para toda la vida, pero muy pocos lo piensan 2 veces, prefieren ir a trabajar y tener plata rápida o ir a afanar y... ir a trabajar para ganar su plata o ir a afanar y tener plata rápida." [Entrevista individual, masculina]

II.c. Influencia de la familia. La familia participa en la construcción de futuro de diferentes formas: como modelos, por sus expectativas sobre el adolescente, ofreciendo alternativas y oportunidades, y orientando al adolescente para que realice en el presente elecciones útiles para alcanzar ese futuro.

"Pero el problema es que si trabajás a los... esto lo pienso yo, más que nada me lo influyó mi viejo que es: vos trabajás a los 15 años y vas a trabajar para toda la vida del mismo oficio, si afanás vas a afanar para toda la vida porque no tenés otra cosa que hacer, no tenés otra fuente de ingreso, de dinero. En cambio si estudiás, tenés eehh... vas a tener que trabajar pero menos o trabajos menos forzosos o podés llegar a estar sentado en una oficina en vez de estar trabajando adentro de una fábrica... que tampoco es algo malo pero es más cómodo" [Entrevista individual masculina].

"yo hablo con mi tío que quiere armar una empresa, algo así. (...) le dije que si necesitaba algo que me llame... y yo iba a seguir 
todo eso de economía. Acá tenés para elegir bachiller y comercial y me dijo que siga comercial y así iba a tener algo en la empresa de él." [Entrevista individual masculina].

"Yo ya de por sí... mi familia está dividida. De parte de mi vieja son todos médicos, dentistas... y del lado de mi viejo son todos abogados, jueces, policías... Yo estoy en el medio, y me dicen: "¿vos que vas a hacer?" [Grupo focal mixto]

II.d. Valoración del proyecto. Las características del proyecto también influyen en las elecciones para el futuro. Los proyectos pueden cambiar en función de la valoración que se hace de la dificultad para alcanzar la meta pero también de la ganancia si estas metas son alcanzadas.

"El problema de ahora es que ponele... el problema de pensar en el futuro, es que vas aprendiendo y vas diciendo lo primero que te gusta. Después no es tanto lo que te gusta, sino es lo que más lo más fácil, lo que te conviene. Después lo que te conviene en el sueldo." [Grupo focal mixto]

Asimismo, pueden cambiar en función de las competencias requeridas para cumplir ese proyecto.

"Porque vos llegás a $1^{\circ}$ y decís: "voy a hacer contador"; y después te dan "Contabilidad" y vos decís: "¿qué m... es esto?" Lo ves re complicado. Yo tengo que aprender esto y tengo que aprender a enseñarlo también" [Grupo focal mixto].

\section{Discusión}

El objetivo del presente trabajo fue conocer cómo percibe un grupo de adolescentes escolarizados de la CABA su futuro y cómo se perciben a sí mismos en él.

En el discurso de los adolescentes aparecieron dos grandes temas: la imagen que los adolescentes tienen de su futuro y la forma en que dicho futuro es construido desde el presente.
Cuando se refieren a su propio futuro o al de sus pares, los adolescentes describen una imagen positiva, que expresa una actitud positiva hacia el futuro. El contenido de dicha imagen hace referencia, en primer lugar, al estudio y la ocupación. En segundo lugar, aparecen la posibilidad de formar una familia y el autosustento. La vocación y ocupación es el contenido típico de los posibles sí mismos en la adolescencia (Marshall, Young, Domene \& Zaidman-Zait, 2008; Oyserman \& James, 2009). También las metas vitales tienen este contenido. Primero aparecen las metas académicas, después las ocupacionales, la familia y, por último, la seguridad material (Massey, Gebhardt, \& Garnefski, 2008). Asimismo, estos son los dominios que se han identificado a través de los países y grupos étnicos en el estudio del contenido temático de la orientación a futuro (Seginer, 2003), lo que implica un contenido transcultural en la valoración que hacen los adolescentes sobre su futuro.

En cuanto a la construcción del futuro, los adolescentes señalan la importancia de trabajar para construir el futuro hoy. Consideran que tienen un rol activo en esa construcción. Este rol está representado, en primer lugar, por el hecho de pensar en el futuro, crear una imagen del futuro y metas que señalan un camino y determinan las acciones que deben realizar en el presente para poder construir el futuro que desean. Lo que los adolescentes refieren como "ocuparse del futuro" se relaciona con los procesos psicológicos involucrados en la orientación hacia el futuro (Nurmi, 1991). Los adolescentes hacen referencia principalmente a la importancia de plantearse metas y planificar su futuro para poder cumplir esas metas. Asimismo, evalúan sus proyectos de acuerdo a sus propios valores (e.g. elegir su profesión por el agrado que piensan que les producirá trabajar en eso o por las posibilidades financieras asociadas a dicha ocupación) y de sus competencias para poder alcanzarlos (e.g. desechar una orientación vocacional por considerar que es demasiado difícil para ellos). La valoración que hace el adolescente de su proyecto y de sus competencias influye en cómo va construyendo su imagen de futuro y sus metas como un proceso dinámico. Todo esto lleva a que para algunos adolescentes dicha imagen sea más clara y definida y para otros sea más vaga, incierta y cambiante. 
En segundo lugar, los adolescentes señalan que la importancia que se otorga a la escuela y el estudio, y el compromiso que se tiene con ellos, es determinante de sus logros futuros. Sus conductas con relación a la escuela y el estudio son las principales acciones que realizan para construirlo desde el hoy. La escuela y las actividades extracurriculares son espacios de capacitación y formación que permiten alcanzar las metas propuestas. Las oportunidades capitalizadas durante la escuela secundaria trazan la diferencia en las aspiraciones, y la construcción y la consecución de metas vitales. Desde la percepción de los adolescentes, las herramientas que otorga la escuela son claves para desenvolverse en el mundo adulto.

La percepción de los adolescentes en relación a la importancia de la escuela y las actividades extracurriculares puede ser vinculada con los conceptos de instrumentalidad y conexión. La conexión como la habilidad para poder relacionar las actividades presentes con las metas futuras. Es una preocupación o falta de preocupación por planificar el futuro (Husman \& Shell, 2008). Los adolescentes hacen énfasis en la importancia de planificar su futuro como una forma de alcanzar sus objetivos y tener mejores posibilidades (e.g. un trabajo mejor). La instrumentalidad constituye el aspecto cognitivo de la perspectiva de tiempo futuro y es definida como la disposición para captar las consecuencias a largo plazo del comportamiento actual (de Volder \& Lens, 1982). Ellos perciben que el compromiso con la escuela y el estudio tienen fuertes consecuencias para su vida futura.

Desde la percepción de los adolescentes que participaron en este estudio, la familia también cumple una función en la construcción del futuro, pero desempeña roles más variados que la escuela: orientar al adolescente en sus elecciones, ser modelos, ofrecer alternativas y oportunidades 0 influir en las elecciones de los adolescentes por sus expectativas sobre ellos. Estas funciones que los adolescentes atribuyen a sus familias se relacionan con los hallazgos respecto de los posibles sí mismos. En estudios previos se encontró que los posibles sí mismos son influenciados por las expectativas de las personas significativas (Oyserman \& Fryberg, 2006), los ideales que los padres transfieren a los hijos (Zentner \& Renaud, 2007), su función como modelos para los adolescentes (Oyserman \& James, 2011). La orientación al futuro también está afectada por la familia, en especial los padres. Tanto los estilos de crianza como las creencias y expectativas de los padres transferidas a sus hijos inciden en su orientación hacia el futuro (Nurmi, 1991; Seginer, 2003).

Un tema emergente en el discurso de los adolescentes es la diferencia que expresan entre la forma en que perciben su futuro o el de sus pares, y el de los adolescentes en general. Cuando se trata de ellos mismos o sus pares más cercanos el futuro es valorado positivamente, su contenido se centra en un desarrollo positivo en distintas áreas, es construido activamente por el adolescente a través de su planificación, el compromiso con la escuela, el estudio y actividades extracurriculares. En cambio "los otros adolescentes" van a tener un futuro negativo, caracterizado por la adicción, la falta de desarrollo personal ("no van a ser nada") o incluso la muerte. Refieren que los otros adolescentes no construyen su futuro porque no piensan en él, viven el presente y no se interesan por la escuela y el estudio.

En consonancia con estos resultados, en un estudio con jóvenes argentinos, se encontró que además de distinguirse a sí mismos y a su grupo de pertenencia de otros grupos con características antagónicas o disímiles también se diferencian de aquellos aspectos que rechazan (Seidman, Azzollini, Thomé y Di lorio, 2011). Es decir, es posible que en el proceso de construir su identidad, tanto actual como futura, los adolescentes busquen diferenciarse de aquellos adolescentes que representan una imagen de la adolescencia que ellos rechazan.

En la lectura que la persona hace del mundo y de su inserción en él, de quién es, a qué contexto pertenece y a cuál no, quiénes son sus pares y cuál resulta ser el rol esperable para él en ese contexto y ese momento específico, se juega tanto la autopercepción como el conjunto de creencias y expectativas que se crean en torno al estereotipo al cual esa persona podría pertenecer o pertenece (Schmidt \& Giménez, 2014). 
En función de esto cabe preguntarse cuán influenciada está la imagen que tienen los adolescentes del futuro de "los otros adolescentes" por la imagen estereotipada de los adultos respecto de la adolescencia. En un estudio realizado en nuestro contexto se encontró que la imagen que tienen los adultos de los adolescentes difiere de la forma en que estos últimos se describen a sí mismos. Los adultos los presentan con mayores problemas emocionales, cambios de humor, y menor capacidad para controlar sus impulsos que la que los adolescentes refieren tener. También sostienen que los adolescentes tienen dificultades para llegar a un acuerdo y resolver conflictos consensuadamente. Se los percibe poco francos y poco dispuestos a colaborar. Los ven menos responsables y menos dedicados, de lo que los adolescentes se perciben. Se apunta a un bajo nivel de autocontrol, describiéndolos como poco confiables (Giménez, 2014). Este estereotipo alimenta un prejuicio negativo en tanto pone de relieve características de personalidad no deseables para la interacción social: la inestabilidad emocional, ser poco responsables y dedicados, con bajos niveles de auto-control e impulsivos, poco francos y sinceros.

\section{Limitaciones y direcciones futuras}

Con respecto a la metodología utilizada, en el enfoque cualitativo se busca profundidad conceptual. Se espera que futuras investigaciones promuevan una transferibilidad de los resultados aquí encontrados a otras unidades de análisis, para lograr una teoría comprensiva formal. En este estudio se trabajó con un grupo particular de adolescentes: todos ellos asistían a tercer año de una escuela pública. Futuras investigaciones deberían indagar esta cuestión y considerar la dimensión de la edad: ¿difiere la percepción y construcción del futuro en adolescentes que inician el secundario respecto de aquellos que lo están terminando? También es de interés el abordaje de este tema considerando las diferencias de género. Los adolescentes de este estudio refieren que la percepción y construcción del futuro difiere en función del género pero en este estudio no se han encontrado esas diferencias en los dichos de los adolescentes.
Por último, resulta relevante comparar las concepciones sobre el futuro de adolescentes de diferentes estratos socioeconómicos así como de aquellos que no están escolarizados.

A su vez, se podrían adoptar enfoques mixtos, utilizando una triangulación de datos cualitativos y cuantitativos para complementar el abordaje de esta compleja temática (Velandia, 2008).

\section{Implicancias prácticas del estudio}

Este trabajo permitió avanzar en la comprensión respecto de cómo un grupo de adolescentes escolarizados de la CABA perciben su futuro y se perciben a sí mismos en él. Conocer la imagen que los adolescentes tienen de su futuro tiene una importancia capital en tanto esta imagen juega un rol significativo en la motivación (Husman \& Lens, 1999) y autorregulación del comportamiento (McInerney, 2004).

Asimismo ha permitido seguir problematizando el estereotipo adolescente, en tanto esta creencia generalizada y exagerada de "los otros adolescentes” está presente en el discurso de los adolescentes para conceptualizar su futuro. Cuando se indaga sobre la fuente de estas creencias estereotipadas sobre la adolescencia, encontramos que los medios de comunicación y los adultos tienen un rol muy importante en la pregnancia de estas ideas. ¿Ofrecemos los adultos modelos alternativos de identificación para la adolescencia? ¿Nos molestamos siquiera en conocerlos, antes de vehiculizar una idea generalizada sobre la adolescencia? En función de los resultados hallados, encontramos que para la construcción de futuro, los nichos que propiciamos los adultos son de gran influencia y permiten el despliegue del repertorio de posibilidades del futuro del adolescente. Esa responsabilidad ha de ponerse de manifiesto, ya que los adolescentes no transitan solos en el devenir a la adultez.

\section{Referencias}

Bembenutty, H. \& Karabenick, S.A. (2004). Inherent association between academic delay of gratification, future time perspective, 
and self-regulated learning. Educational Psychology Review, 16(1), 35-57. doi. org/10.1023/B:EDPR.0000012344.34008.5c

Blakemore, S.J. \& Choudhury, S. (2006). Development of the adolescent brain: implications for executive function and social cognition. Journal of Child Psychology and Psychiatry, and Allied Disciplines, 47(3-4), 296-312. doi. org/10.1111/j.1469-7610.2006.01611.x

De Volder, M.L. \& Lens, W. (1982). Academic achievement and future time perspective as a cognitive-motivational concept. Journal of Personality and Social Psychology, 42(3), 566571. doi.org/10.1037/0022-3514.42.3.566

Erikson, E.H. (1968). Identidad, juventud y crisis. Buenos Aires: Paidós.

Erikson, M.G. (2007). The meaning of the future: Toward a more specific definition of possible selves. Review of General Psychology, 11(4), 348-358. doi.org/10.1037/1089-2680.11.4.348

Giménez, M. (2014). Evaluación de características de la personalidad adolescente: autopercepción y estereotipo. Buenos Aires: Universidad de Buenos Aires.

Hernández, R., Fernández-Collado, C. \& Baptista, P. (2010). Metodología de la investigación [Research methodology] (5ta ed.). México: Mc Graw Hill.

Husman, J. \& Lens, W. (1999). The role of the future in student motivation. Educational Psychologist, 34(2), 113-125. doi.org/10.1207/ s15326985ep3402_4

Husman, J. \& Shell, D.F. (2008). Beliefs and perceptions about the future: A measurement of future time perspective. Learning and Individual Differences, 18(2), 166-175. doi.org/10.1016/j. lindif.2007.08.001

Krauskopf, D. (2003). Proyectos, incertidumbre y futuro en el período juvenil. Archivo Argentino de Pediatría, 101(6), 495-500. Retrieved from http://www3.sap.org.ar/staticfiles/archivos/2003/arch03_6/A6.495-500.Krauskopf.pdf
Markus, H. \& Nurius, P. (1986). Possible Selves. American Psychologist, 41(9), 954-969. doi. org/10.1037/0003-066X.41.9.954

Marshall, S.K., Young, R.A., Domene, J. F., \& Zaidman-Zait, A. (2008). Adolescent possible selves as jointly constructed in parentadolescent career conversations and related activities. Identity: An International Journal of Theory and Research, 8, 185-204. doi. org/10.1080/15283480802181727

Massey, E.K., Gebhardt, W.A. \& Garnefski, N. (2008). Adolescent goal content and pursuit: $A$ review of the literature from the past 16 years. Developmental Review, 28, 421-460. Retrieved from http://www.sciencedirect.com/science/ article/pii/S0273229708000166

Maxwell, J. (2012). Qualitative research design: An interactive approach (3era ed.). London: SAGE Publications.

Mclnerney, D.M. (2004). A Discussion of future time perspective. Educational Psychology Review, 16(2), 141-151. doi.org/10.1023/ B:EDPR.0000026610.18125.a3

Miller, R.B. \& Brickman, S.J. (2004). A model of future-oriented motivation and self-regulation. In Educational Psychology Review, 16, 9-3). doi. org/10.1023/B:EDPR.0000012343.96370.39

Nurmi, J. (1991). How do adolescents see their future? A review of the development of future orientation and planning. Developmental Review, 11(1), 1-59. doi.org/10.1016/0273-2297(91)90002-6

Oyserman, D. (2001). Self-concept and identity. In A. Tesser \& N. Schwarz (Eds.), Blackwell handbook of social psychology: Intraindividual processes (pp. 499-517). Massachusetts, CA: Blackwell.

Oyserman, D. \& Fryberg, S. (2006). The possible selves of diverse adolescents: Content and function across gender, race and national origin. In C. Dunkel \& J. Kerpelman (Eds.), Possible selves: Theory, research, and applications (pp. 1-23). New York: Nova Science Publishers. 
Oyserman, D. \& James, L. (2009). Possible selves: From content to process. In K. D. Markman, W. M. Klein \& J. A. Suhr (Eds.), Handbook of imagination and mental simulation (pp. 373-394). New York, NY: Taylor \& Francis.

Oyserman, D. \& James, L. (2011). Possible identities. In S.J. Schwartz, K. Luyckx \& V.L. Vignoles (Eds.), Handbook of Identity Theory and Research (pp. 117-145). New Jersey: Springer Link. doi.org/10.1007/978-1-4419-7988-9

Oyserman, D. \& Markus, H. (1990). Possible selves and delinquency. Journal of Personality and Social Psychology, 59(1), 112-125. Retrieved from http://www.ncbi.nlm.nih.gov/ pubmed/2213484

Phan, H.P. (2009). Future time perspective in sociocultural contexts: A discussion paper. Electronic Journal of Research in Educational Psychology, 7(2), 761-778. Retrieved from http://www.investigacion-psicopedagogica.org/revista/articulos/18/english/Art_18_340.pdf

Piñuel, J. (2002). Epistemología, metodología y técnicas del análisis de contenido. Estudios en Sociolinguística, 3(1), 1-42.

Schmidt, V. \& Giménez, M. (2014). Consumo de alcohol en la adolescencia: el rol del estereotipo adolescente. En Memorias del VI Congreso Internacional de Investigación y Práctica Profesional en Psicología y XXI Jornadas de Investigación de la Facultad de Psicología de la Universidad de Buenos Aires. Buenos Aires, Argentina: Universidad de Buenos Aires. Facultad de Psicología. Secretaría de Investigaciones.
Seginer, R. (2003). Adolescent Future Orientation: An Integrated Cultural and Ecological Perspective. Online Readings in Psychology and Culture, 6, 1-13. Retrieved from http://scholarworks.gvsu. edu/orpc/vol6/iss1/5/

Seidman, S., Azzollini, S., Thomé, S. y Di lorio, J. (2011). Construcciones identitarias, juventud y vida cotidiana: Un estudio desde la teoría de las representaciones sociales. Anuario de Investigaciones de La Facultad de Psicología de La Universidad de Buenos Aires, XVIII, 295-300. Recuperado de http://www.scielo.org.ar/scielo.php?script=sci_arttext\&pid $=$ S1851-16862011000100032

Stouthard, M.E. \& Peetsma, T.T. (1999). FutureTime Perspective: Analysis of a FacetDesigned Questionnaire. European Journal of Psychological Assessment, 15(2), 99-105. doi. org/10.1027//1015-5759.15.2.99

Strauss, A. \& Corbin, J. (1990). Basics of qualitative research: Grounded Theory procedures and techniques. London SAGE Publications.

Velandia, A. (2008). Investigación cualitativa y psicología del consumidor: alternativas de aplicación. Avances en Psicología Latinoamericana, 26(2), 290-303.

Zentner, M. \& Renaud, O. (2007). Origins of adolescents' ideal self: an intergenerational perspective. Journal of Personality and Social Psychology, 92(3), 557-574. doi. org/10.1037/0022-3514.92.3.557 